\title{
COVID-19 Exposure, Stress, and
} Mental Health Outcomes: Results From a Needs Assessment Among Low Income Adults in Central North Carolina

\author{
Andréa R. Kaniuka ${ }^{1}$, Robert J. Cramer ${ }^{1 *}$, Corrine N. Wilsey ${ }^{1}$, \\ Jennifer Langhinrichsen-Rohling ${ }^{2}$, Annelise Mennicke ${ }^{3}$, Alexandra Patton ${ }^{1}$, \\ Meagan Zarwell ${ }^{1}$, Carmen P. McLean ${ }^{4,5}$, Yu-Jay Harris ${ }^{6}$, Sharon Sullivan ${ }^{6}$ and Glori Gray ${ }^{6}$ \\ ${ }^{1}$ Department of Public Health Sciences, University of North Carolina at Charlotte, Charlotte, NC, United States, ${ }^{2}$ Department \\ of Psychological Sciences, University of North Carolina at Charlotte, Charlotte, NC, United States, ${ }^{3}$ School of Social Work, \\ University of North Carolina at Charlotte, Charlotte, NC, United States, ${ }^{4}$ National Center for PTSD, Dissemination and \\ Training Division, VA Palo Alto Health Care System, Menlo Park, CA, United States, ${ }^{5}$ Department of Psychiatry and \\ Behavioral Sciences, Stanford University, Stanford, CA, United States, ${ }^{6}$ Psychology for All, Charlotte, NC, United States
}

OPEN ACCESS

Edited by:

Kairi Kölves,

Griffith University, Australia

Reviewed by:

Eleonora Topino,

Assunta University, Italy

Angela Jayne Clapperton,

The University of Melbourne, Australia

*Correspondence:

Robert J. Cramer

rcramer4@uncc.edu

Specialty section: This article was submitted to Public Mental Health a section of the journal

Frontiers in Psychiatry

Received: 06 October 2021 Accepted: 25 November 2021

Published: 20 January 2022

Citation:

Kaniuka AR, Cramer RJ, Wilsey CN, Langhinrichsen-Rohling J, Mennicke A, Patton A, Zarwell M, McLean CP, Harris Y-J, Sullivan S and Gray G (2022) COVID-19 Exposure, Stress, and Mental Health Outcomes:

Results From a Needs Assessment Among Low Income Adults in Central North Carolina.

Front. Psychiatry 12:790468. doi: 10.3389/fpsyt.2021.790468
This study focuses on identifying COVID-19 related exposure, stress, and mental health concerns in the larger Charlotte, North Carolina region, an area with many low-income and under resourced communities. A community-academic partnership conducted a regional COVID-19 needs assessment. Low-income adults $(N=156)$ completed an online-administered survey of demographic information, COVID-19 exposure, stress, coping-related factors, and mental health. Frequency data showed that common COVID-19 related stressors included job exposure, lost job/income, and increased home responsibilities. Frequency data further showed elevated screening risk rates for mental health concerns were observed for post-traumatic stress (83.3\%), depression (52.2\%), problematic drinking (50.0\%), generalized anxiety (43.0\%), and suicide (40.4\%). Bivariate correlation and multivariate regression models identified robust mental health risk factors including COVID-19 related stress affecting close persons, fear/worry reaction to the pandemic, and use of venting as a coping strategy; protective factors included active coping and problem-focused coping beliefs. Findings are discussed with respect to informing regional public health efforts during the pandemic.

Keywords: COVID-19, coping, stress, mental health, suicide

In January 2020, the novel coronavirus (COVID-19) was declared a public health emergency in the United States after the World Health Organization reported the spread of the virus as an international problem (1). As of September 2021, an estimated 220 million cases were documented worldwide, including over 650,000 deaths in the United States alone (2). In addition to proximal COVID-19 symptoms and death, the pandemic and associated public health interventions (e.g., home-confinement, social distancing) have resulted in psychosocial and mental health impacts (3). As such, the International COVID-19 Suicide Prevention Research Collaboration released a global call to action anticipating rising adverse mental health effects due to the ongoing pandemic (4). The present study contributes to that call through a focus on vulnerable, low-income adults in an urban region of the southern United States. 


\section{WHAT DO WE KNOW ABOUT STRESS, BEHAVIORAL RESPONSES, AND MENTAL HEALTH IN PANDEMICS?}

Globally, rates of mental disorders (e.g., depression, anxiety, post-traumatic stress disorder) are a concern, with a prevalence of approximately $22 \%$ (5); further, low-income individuals are at increased risk of mental health disorders and suicide attempts (6). Public health emergencies may exacerbate rates of negative mental health outcomes. Individuals and communities experiencing public health emergencies may endure a number of emotional and behavioral stressors and behavioral impacts, including increased distress or mental health conditions, and increased substance use (7). For example, survey data suggests that quarantine, isolation, and other social distancing measures during pandemic-focused disasters are associated with stress and the risk for post-traumatic stress disorder (8). In Singapore, one study identified increased rates of psychiatric symptoms (e.g., post-traumatic effects) ranging between 22.9 and $25.8 \%$ among the general population (9). The long-term mental health outcomes following the 2009 influenza strain H1N1 (i.e., swine flu) pandemic included increased rates of anxiety, depression, and a greater risk for post-traumatic stress disorder (10).

Recent studies similarly indicate that poor mental health outcomes may be associated with the ongoing COVID-19 pandemic $(3,11,12)$. Globally, rates of mental health issues, including depression, anxiety, and post-traumatic stress (PTS) increased during the initial months of the COVID-19 pandemic compared to pre-pandemic rates $(13,14)$. Further, besides PTS, the pandemic has been linked with increased feelings of apprehension, boredom, anger, fear, uncertainty, loneliness, stress, anxiety, and suicide ideation (14-17). For instance, interviews with college students in the United States showed an escalation in rates of stress and anxiety during the pandemic (18). Both the virus and associated safety guidelines represent stressors, with extended periods of social distancing straining mental health $(15,19)$. For example, findings early on in the pandemic (March 2020), suggested that social distancing was linked with worse mental health (e.g., symptoms of depression, anxiety, insomnia, and acute stress) (20). Other documented stressors associated with the pandemic include financial difficulties, fear of becoming physically ill, and concern about accessing mental health resources (21-24).

Repeated exposure to global media coverage related to the virus and social distancing can also perpetuate stress and anxiety and influence noncompliance of public health directives (e.g., mask mandates), leading to absence of help-seeking behaviors (25). In terms of reactions to the pandemic and its media coverage, research conducted in multiple countries demonstrates adverse mental health impacts including sleep disturbance, attention difficulties, extreme increased substance use, less social interaction, and increased social isolation, as well as lowered perceived physical health $(18,26)$.

Understanding how these stressors interact with known social risk factors is critical to understanding the mental health impact of the pandemic. Studies of the impact of COVID-19 on mental health have largely focused on samples of convenience or have focused on specific occupational groups (e.g., medical staff) or medically vulnerable populations. However, low socioeconomic status is a critical social risk factor that may heighten the risk for negative mental health outcomes, warranting research with low socioeconomic status populations. For example, longitudinal research indicates that overall depressive symptoms among U.S. adults increased from early in the pandemic compared to 1 year later, with low household income exacerbating depressive symptoms (27). An important aim of the present study is to examine a range of stressors attributed to COVID19 among individuals who are disproportionately affected by socioeconomic systems that lead to poverty.

Emerging evidence suggests the pandemic may result in both helpful and unhelpful coping strategies (e.g., venting, active coping, substance use) $(23,24)$. Documented efforts to cope with pandemic-related stress include positive (e.g., social support, mindfulness) and negative (e.g., substance use, social withdrawal) coping techniques $(28,29)$. For example, healthy (e.g., mindfulness) and unhealthy (e.g., sleeping longer) selfmanagement strategies and seeking social support were common among college students grappling with the pandemic (18). An additional aim of this study is to quantify COVID-19 related exposure and stress, especially as they relate to the mental health and coping of adults with lower incomes in the Southern United States.

\section{WHY CENTRAL NORTH CAROLINA?}

Central North Carolina (NC), including Charlotte and its surrounding areas, has a deep history of social and economic inequality. Approximately $14 \%$ of the region lives both below the poverty line and without health insurance (30). More than $50 \%$ of the region are people of color, with over $30 \%$ identifying as African American and another 14\% as Hispanic. In addition, at least $15 \%$ are immigrants and refugees. Thus, a substantial proportion of the population is exposed to socioeconomic systems that lead to poverty, institutional racism, and discrimination that targets Black, Indigenous People of Color (BIPOC), and socio-political policies that target undocumented immigrants, all of which may heighten the negative impact of the pandemic on mental health and/or poor coping strategies. Indeed, findings show that the COVID-19 pandemic has disproportionately affected members of low-income and urban communities of color $(31,32)$, likely exacerbated by preexisting and ongoing social, health, and economic inequities. The demographic diversity of central NC leaves the area particularly vulnerable to long-lasting negative impacts of COVID-19. In NC, more than 31,000 people are estimated to be at risk of complications due to COVID-19, with patterns worse for racially and ethnically minoritized groups and those without health insurance (33). BIPOC individuals are more likely to have preexisting conditions such as asthma or diabetes, and are more likely to work jobs deemed essential, increasing their likelihood of exposure to SARS-CoV-2, and ultimately leading to higher rates of COVID-19 mortality $(34,35)$. The demographic, financial, 
and social composition of central NC raises concern about the long-term impact of the local COVID-19 pandemic.

The broader public health research literature helped us identify starting points for this COVID-19 needs assessment. For instance, a June 2020 CDC study regarding mental health during the pandemic showed that $40 \%$ of those surveyed reported struggling with mental health or substance use (36). Specifically, $31 \%$ of individuals reported symptoms of anxiety/depression, $26 \%$ indicated having a trauma-related concern, $13 \%$ started or increased substance use, and $11 \%$ considered suicide. Further, substance abuse and suicidal ideation frequencies were higher among young adults and members of racial/ethnic minority groups. The COVID-19 pandemic has resulted in the publication and dissemination of numerous professional organization resource pages on stress, coping, and related matters $(37,38)$. However, studies that evaluate both COVID-19-related stress and exposure, as well as coping and biopsychosocial responses, are needed to understand the mental health needs of low socioeconomic status adults in the central NC region. Doing so offers the possibility of developing a tailored intervention program specific to the region's needs.

\section{THE PRESENT STUDY}

Groups that have been economically and socially marginalized in the central NC region may disproportionately experience stress, negative reactions, and psychological disruptions caused by COVID-19. Among low-income adults in the central NC region, the aims of this community-engaged needs assessment were:

Aim 1: To quantify COVID-19 specific exposure, stress, and responses.

Aim 2: To assess levels of mental health concerns and coping strategies and beliefs.

Aim 3: To identify COVID-19 and coping-related risk and protective factors for mental health outcomes.

\section{MATERIALS AND METHODS}

\section{Community-Engaged Partnership}

This study was conducted with help from a communityacademic partnership (39). Psychology for All (40) is a Charlotte area non-profit aimed at reducing barriers to mental health services for people with lower incomes. The university team comprised community-focused researchers in public health, psychology, and social work. Consistent with communityacademic partnership principles (39), problem identification was aimed at helping solve pandemic and health-related problems of interest to constituent partners. This project served as an initial needs assessment to inform community action and public health program development.

\section{Procedure}

University investigators obtained Institutional Review Board approval for this assessment. Data were collected in late July of 2020. Convenience sampling was used via an online Qualtricsadministered self-report survey constructed for distribution by
Psychology for All (40) and its constituent community partners via email listserv and social media distribution. Importantly, the following partners all serve ethnic, racial and/or sexual minority groups and people experiencing poverty. We engaged with these partners specifically to reach groups disproportionately experiencing health disparities in Charlotte, NC. Partners sharing the survey opportunity were C4 Counseling, The Harvest Center, Care Ring, Time Out Youth, Westside Education Think Tank, and UNC Charlotte School of Social Work field education partners (41-46). Psychology for All distributed a standard email advertisement of the survey to individual administrative contacts at each constituent partner. Partners shared the email advertisement, which included study goals, participant risks/benefits and the survey link, to their respective clientele. Response rate could not be tabulated because partners did not report back overall listserv sizes.

The informed consent contained information about the study aims, procedure, investigators and contact information, participant rights, and renumeration details. Potential participants indicated consent by selecting "yes" for their willingness to participate in the online consent form. Potential participants first completed the study screener to confirm study eligibility criteria of (1) 18 years of age or older, (2) annual household income of $\$ 60,000$ or less, and (3) resident in Charlotte-Mecklenburg or surrounding counties (i.e., Gaston, Lincoln, Cabarrus, and Union). The screener survey immediately ended if a potential participant did not meet study inclusion criteria. All subsequent measures (see below) were presented in randomized order, so as to avoid response set effects. Information regarding Psychology for All's online therapy service application, Psychology Today's mental health provider locator, and national crisis phone and text line were given on each survey page and in consent/debriefing documents. Upon survey completion, participants were provided with a written debriefing page. They were also offered the opportunity to provide an email address and preference for a $\$ 20.00$ Amazon or Walmart e-gift card. The survey took approximately 15-20 min to complete.

\section{Participants}

Online Supplement 1 contains a demographic summary. Participants $(N=156)$ were primarily from Charlotte/Mecklenburg or Lincoln County and were born in the United States. Most participants were either White or Black/African American, between 36 and 55 years of age $(n=91$; $58.3 \%)$, and identified evenly as either men $(n=79 ; 51.0 \%)$ or women $(n=75 ; 48.4 \%)$. Further, the majority of participants were employed either full or part-time, had an Associate's degree or less, and were insured.

\section{Measures \\ Demographics}

A demographics form first screened participants for age, annual household income, and county of residence, followed by gender, race, ethnicity, insurance status, education level, employment status, and whether the person had been advised to see a mental health provider. 


\section{COVID-19 Exposure, Stress and Responses}

COVID-19 exposure and adjustment measures developed for community research use by the Department of Veterans Affairs were used in the present study. The Coronavirus Stress Survey (47) comprises 10 binary response (i.e., no/yes) questions capturing virus exposure, illness, and difficulties (e.g., medical challenges, familial responsibilities) associated with COVID-19, as well as COVID-19 media exposure. Respondents indicated whether the events happened to themselves and/or someone they know. A total score was tabulated each for personal and other known person stressors. Internal consistency values (Cronbach's $\alpha$ ) for the respective COVID-10 stress self (0.54) and other (0.56) values were low. The Coronavirus Response Scale-10 (CRS-10) (48) assesses specific domains of impact such as pain, social support, physical activity, and emotional distress. Items are scored and used individually.

\section{Depressive Symptoms}

The Patient Health Questionnaire-2 (PHQ-2) (49) is a twoitem screening tool used to assess depressed mood and little interest/pleasure in activities. Items are indicated on a 4-point Likert scale ranging from 0 (not at all) to 3 (nearly every day). Items are summed to provide both total and cut scores $(>3)$ to identify those at risk for severe depression. Internal consistency (Cronbach's $\alpha$ ) in the present sample was 0.64 . As a screening tool for major depressive disorder, the PHQ-2 cut off score demonstrates high sensitivity (83\%) and specificity (92\%). Additionally, scores on the PHQ-2 are associated with mental health and social and physical functioning (49).

\section{Anxiety Symptoms}

The Generalized Anxiety Disorder-7 (GAD-7) (50) is a seven-item questionnaire capturing seven domains of anxiety symptoms (e.g., feeling nervous, worrying). Items are indicated on a 4-point Likert scale ranging from 0 (not at all) to 3 (nearly every day). Responses are summed for a total score, which is subsequently classified into four severity categories and a clinical cut-off score $(\geq 10)$. Internal consistency (Cronbach's $\alpha$ ) for the total score in the present sample was 0.79. The GAD7 demonstrates excellent internal consistency (Cronbach's $\alpha$ ) among general population samples $(\alpha=0.92)$ and the sensitivity $(89 \%)$ and specificity ( $82 \%)$ of the clinical cut-off are high. Scores on the GAD-7 are significantly positively correlated with other anxiety scales, including the Beck Anxiety Inventory (50).

\section{Suicide Risk}

The Suicidal Ideation Attributes Scale (SIDAS) (51) contains five items assessing suicidal thinking, controllability, and impact. Items are indicated on a variably anchored 11-point Likert scale. Responses are summed to provide a total and cut score ( $>21)$ for identification of those at risk for suicide. The SIDAS demonstrates excellent internal consistency among community samples $(\alpha=0.91)$ and the cut-off score has excellent specificity in identifying individuals at risk of suicidal behavior. Internal consistency (Cronbach's $\alpha$ ) in the present sample was 0.92. Additionally, items on the SIDAS are significantly positively associated with other measures of suicidal ideation, such as the Columbia Suicide Severity Scale, as well as measures of depression (PHQ-9) and anxiety (GAD-7) (51).

\section{Post-traumatic Stress Disorder (PTSD) Symptoms}

The Post-traumatic Checklist-2 (PCL-2) (52-54) is a twoitem screener of key post-traumatic symptoms (e.g., intrusive thoughts/images). The 2 -item version of the PCL contains the two items from the longer PCL- 6 that were most correlated with total score (53). Responses are indicated on a 5-point Likert scale ranging from 1 (not at all) to 5 (extremely). Responses are summed to provide a total score and cut score $(\geq 4)$ for identifying probable PTSD diagnosis. Internal consistency (Cronbach's $\alpha$ ) for the total score in the present sample was 0.69 . The sensitivity of the PCL-2 cutoff in identifying individuals with PTSD is high (0.97) (52).

\section{Problematic Drinking}

The Alcohol Use Disorders Identification Test-C (AUDIT-C) (55) is a three-item inventory assessing problematic alcohol use (e.g., frequency of binge drinking). Items are indicated on a 5-point variably anchored Likert scale ranging from 0 to 4 . Responses are summed, providing a summed score and cutscore ( $\geq 4$ for men; $\geq 3$ for women) to identify individuals at risk for alcohol abuse. Internal consistency (Cronbach's $\alpha$ ) for the total score in the present sample was 0.52. The AUDIT$\mathrm{C}$ is significantly correlated with other measures of hazardous drinking (56). Further, the AUDIT-C demonstrates acceptable internal consistency ( $\alpha=0.70)$ (57) and is effective in screening for alcohol abuse/dependence (sensitivity $=0.88)(55)$.

\section{Coping and Resilience}

The Brief COPE (58) is a 28 -item measure containing 14 coping style subscales. We selected three items to assessing venting, active coping, and reframing coping styles. As indicated in the original publishing of the scale, the full instrument does not need to be administered and items and scales can be selected for administration (58). Internal consistency (Cronbach's $\alpha$ ) values for Brief COPE subscales used in the present sample were 0.62 for active coping, 0.23 for venting, and 0.59 for positive reframing. The Coping Self-Efficacy Scale (59) was used to assess three areas of beliefs about one's ability to use the following coping strategies: thought stopping, problem-focused coping, and getting social support. Items are indicated on an 11-point Likert scale ranging from 0 (I cannot do this at all) to 10 (I'm certain that I can do this) and summed for a total score. Internal consistency of the CSE subscales is good (thought stopping: $\alpha=0.91$, problem-focused coping: $\alpha=0.91$, getting social support: $\alpha=0.80$ ). Additionally, CSE subscales are associated with measures of psychological distress (e.g., anxiety, perceived stress), well-being (e.g., optimism), social support, and ways of coping. CSE subscale internal consistency values (Cronbach's $\alpha$ ) in the present sample were 0.92 for problem-focused coping, 0.91 for thought stopping, and 0.93 for getting social support. Finally, the Connor-Davidson Resilience Scale-2 item (CD-RISC2) (60) served as a screener for participants' ability to bounce back from difficult circumstances. The CD-RISC2 is significantly negatively correlated with perceived stress and with overall score on the 
TABLE 1 | Correlation matrix of COVID-19 related stress and responses, coping factors, and mental health outcomes.

\begin{tabular}{|c|c|c|c|c|c|c|}
\hline MH correlate & Depression & SI & PTS & Anxiety & Alcohol use & $\alpha$ \\
\hline COVID-19 stress-self total & 0.16 & -0.08 & 0.15 & 0.03 & 0.01 & 0.56 \\
\hline COVID-19 stress-other total & 0.32 & 0.37 & 0.21 & 0.30 & 0.46 & 0.54 \\
\hline CRS-10 stress response & 0.17 & -0.21 & 0.26 & 0.12 & -0.09 & - \\
\hline CRS-10 interaction with friends and family & -0.02 & -0.03 & -0.08 & -0.24 & -0.02 & - \\
\hline CRS-10 emotional distress response & 0.31 & 0.04 & 0.21 & 0.17 & 0.10 & - \\
\hline CRS-10 physical activity & 0.18 & 0.32 & 0.09 & 0.18 & 0.18 & - \\
\hline CRS-10 use of alcohol and illicit drugs & 0.37 & 0.36 & 0.35 & 0.35 & 0.42 & - \\
\hline CRS-10 use of prescription medication & 0.21 & 0.43 & 0.22 & 0.23 & 0.26 & - \\
\hline CRS-10 pain & 0.33 & 0.34 & 0.36 & 0.29 & 0.25 & - \\
\hline CRS-10 fear or worry & 0.28 & 0.03 & 0.43 & 0.36 & 0.15 & - \\
\hline CRS-10 effort to cope with stress & 0.19 & 0.04 & 0.26 & 0.14 & 0.01 & - \\
\hline CRS-10 overall sense of well-being & 0.02 & 0.12 & 0.12 & 0.01 & 0.14 & - \\
\hline Brief resilience & -0.14 & -0.29 & -0.16 & -0.29 & -0.22 & 0.73 \\
\hline Active coping & -0.06 & -0.43 & -0.03 & -0.11 & -0.29 & 0.62 \\
\hline Venting & 0.23 & 0.25 & 0.34 & 0.31 & 0.13 & 0.23 \\
\hline Positive reframing & -0.10 & -0.29 & -0.09 & -0.07 & -0.15 & 0.59 \\
\hline Problem-focused coping beliefs & -0.26 & -0.40 & -0.13 & -0.33 & -0.25 & 0.92 \\
\hline Thought stopping beliefs & -0.15 & -0.24 & -0.05 & -0.17 & -0.05 & 0.91 \\
\hline Getting social support beliefs & -0.09 & -0.30 & -0.04 & -0.14 & -0.08 & 0.93 \\
\hline$\alpha$ & 0.65 & 0.91 & 0.69 & 0.79 & 0.52 & - \\
\hline
\end{tabular}

Bold font denotes $p<0.001$; Bold italics font $p<0.05$.

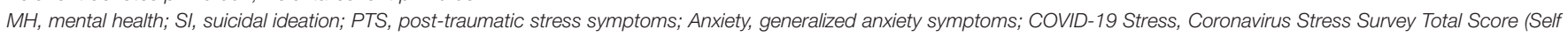
or Other); CRS-10, Coronavirus Response Scale-10.

full CD-RISC (60). Internal consistency (Cronbach's $\alpha$ ) for the

CD-RISC2 total score was 0.73 in the present sample.

Internal consistencies for the current study are reported in Table 1.

\section{Data Analysis}

Aim 1 and Aim 2 analyses were accomplished using simple frequency counts. Total mental health outcome scores were converted to categories using clinical cut-score values summarized in the measures section. Bivariate correlations were used as the first analytic step in Aim 1 in order to identify bivariate risk and protective factors of mental health outcomes. Following guidelines in the literature (61), multivariate regression was then employed to (a) select demographic covariates of mental health outcomes, and (b) identify the most robust risk and protective factors for mental health outcomes.

\section{RESULTS}

\section{COVID-19 Specific Exposure, Stress, and Responses}

Table 2 contains results from the Coronavirus Stress Survey. Less than one-third of participants reported direct exposure or illness, but almost three-quarters reported knowing someone close who was affected by COVID-19. The most common self-reported coronavirus-related stressors were job exposure, lost job/income, and increased home responsibilities. The most common reported stressors for known close persons were lost job/income, increased
TABLE 2 | Self- and other-experienced COVID-19 related exposure and stressors.

\begin{tabular}{lcc}
\hline Coronavirus related experience & $\begin{array}{c}\text { Happened } \\
\text { to me-n (\%) }\end{array}$ & $\begin{array}{c}\text { Happened } \\
\text { to someone } \\
\text { close to } \\
\text { me-n (\%) }\end{array}$ \\
\hline $\begin{array}{l}\text { 1. Become ill from possible or certain exposure } \\
\text { to the coronavirus }\end{array}$ & $46(29.5 \%)$ & $111(71.2 \%)$ \\
$\begin{array}{l}\text { 2. Job requires possible exposure to } \\
\text { coronavirus }\end{array}$ & $73(46.8 \%)$ & $65(41.7 \%)$ \\
$\begin{array}{l}\text { 3. Lost job or lost income due to the } \\
\text { coronavirus pandemic }\end{array}$ & $72(46.2 \%)$ & $84(53.8 \%)$ \\
$\begin{array}{l}\text { 4. Increased responsibilities at home due to the } \\
\text { coronavirus pandemic }\end{array}$ & $73(46.8 \%)$ & $88(56.4 \%)$ \\
$\begin{array}{l}\text { 5. Difficulty getting food, medication or other } \\
\text { necessities due to the coronavirus pandemic }\end{array}$ & $52(33.3 \%)$ & $76(48.7 \%)$ \\
$\begin{array}{l}\text { 6. Difficulty getting needed social support due } \\
\text { to the coronavirus pandemic }\end{array}$ & $64(41.0) \%$ & $71(45.5 \%)$ \\
$\begin{array}{l}\text { 7. Lost health insurance due to the coronavirus } \\
\text { pandemic }\end{array}$ & $40(25.6 \%)$ & $70(44.9 \%)$ \\
$\begin{array}{l}\text { 8. Went on public food assistance due to the } \\
\text { coronavirus pandemic }\end{array}$ & $54(34.6 \%)$ & $62(39.7 \%)$ \\
\hline
\end{tabular}

home responsibilities, and difficulties getting basic necessities (e.g., medication). A total of $83.4 \%$ of the sample reported consuming one or more hours per day of COVID-19 related media information (e.g., TV, Twitter, Facebook). 
TABLE 3 | Mental health and coping-related descriptive statistics.

\begin{tabular}{|c|c|c|c|c|}
\hline $\begin{array}{l}\text { Mental health or coping } \\
\text { related factor }\end{array}$ & $M(S D)$ & Mean score label & $\begin{array}{c}n(\%) \text { above cut score } \\
\text { suggesting increased risk }\end{array}$ & Interpretation of elevated risk \\
\hline Depression & $2.47(1.46)$ & No risk & $83(52.2 \%)$ & Probable risk for depression \\
\hline Suicidal thinking & $14.62(11.50)$ & No risk & $63(40.4 \%)$ & Elevated suicide risk \\
\hline Post-traumatic stress & $5.61(1.82)$ & Possible PTSD & 130 (83.3\%) & Possible PTSD \\
\hline Anxiety & $8.09(4.02)$ & Moderate anxiety & $67(43.0 \%)$ & Moderate or worse \\
\hline Alcohol use - men ${ }^{a}$ & $3.39(1.50)$ & No risk & $40(50.6 \%)$ & Problematic drinking \\
\hline Alcohol use - women & $2.67(1.91)$ & No risk & $38(50.0 \%)$ & Problematic drinking \\
\hline Resilience & $3.38(0.88)$ & Neutral & - & - \\
\hline Active coping & $2.69(0.70)$ & Doing a medium amount & - & - \\
\hline Venting & $2.49(0.70)$ & Doing a little bit & - & - \\
\hline Positive reframing & $2.77(0.75)$ & Doing a medium amount & - & - \\
\hline Problem-focused coping beliefs & $5.24(2.07)$ & Moderately certain I can do this & - & - \\
\hline Thought stopping beliefs & $5.26(2.18)$ & Moderately certain I can do this & - & - \\
\hline Getting social support beliefs & $5.49(2.26)$ & Moderately certain I can do this & - & - \\
\hline
\end{tabular}

M, mean; SD, standard deviation; PTSD, Post-traumatic stress disorder.

a AUDIT-C requires breakdown by gender for use of cut-scores.

A total of 85 participants provided narrative responses regarding additional COVID-19 related concerns. Responses fell into the following categories (a respondent could provide more than one type): job or financial loss $(n=14)$; decreased socialization or being stuck at home $(n=13)$; personal mental health or negative mood $(n=9)$; job pressures $(n=5)$; no school for kids $(n=5)$; know someone who died of COVID-19 $(n=5)$; having to engage in preventive practices (e.g., wearing a mask) $(n=4)$; and child rearing $(n=4)$. Common responses to the Coronavirus pandemic were cataloged using the CRS-10 items (see Table 1 for items). Descriptive patterns were similar for all 10 items, with average scores falling approximately at the mid-point $\left(M_{\text {range }}=2.66\right.$ to $3.24, S D_{\text {range }}=0.96$ to 1.16$)$. These scores reflect responses to the pandemic as "about the same" as compared to before the pandemic.

\section{Mental Health Concerns and Coping-Related Strategies}

Table 3 contains descriptive summaries for mental health outcomes and coping-related scales. Scores on PTSD and generalized anxiety screeners indicate elevated levels among this sample. Clinical cut scores derived from source articles (see measures section) show concerning rates of probable risk (in descending order) for post-traumatic stress, depression, problematic drinking, generalized anxiety, and suicide risk. With the exception of venting, participants reported scale midpoint levels of all coping-related factors. Venting was used less than the midpoint.

\section{COVID-19 and Coping-Related Risk and Protective Factors for Mental Health Outcomes}

Table 1 contains a bivariate correlation matrix of the following factors related to mental health outcomes: (1) all summed
COVID-19 stress survey scores for self and other (both derived from items on Table 2, score range 0-8); (2) HCRS-10 items; and (3) coping-related factors. Correlates most robustly related to better mental health outcomes included resilience and problem-focused coping beliefs. The strongest correlates of negative mental health outcomes were COVID-19 related stress others (Coronavirus Stress Survey-others subscale); responses to the pandemic-physical activity level, pain, alcohol/drug use, prescription medication use, fear/worry (CRS-10 items); and the coping domain of venting.

Prior to evaluating bivariate correlates of mental health outcomes, we sought to identify necessary demographic covariates. Due to low cell sizes in many categories, demographics were recoded for regression analyses: gender ( $1=$ male, $2=$ female [ 1 'other' dropped']), age $(1=18-35$, $2=36+)$, county $(1=$ Charlotte/Mecklenburg [the inner lying urban county], $2=$ other $)$, race $(1=$ White, $2=$ racial minority), ethnicity ( $1=$ Non-Hispanic; $2=$ Hispanic or other minority), education ( $1=$ High school/GED or less, $2=$ Associate's Degree or higher), and employment status ( $1=$ employed, $2=$ unemployed or retired). Binary demographic variables were entered into a multivariate regression model with all mental health outcomes included. Demographics with significant overall multivariate effects on mental health outcomes were retained as control variables for further analysis. Only gender (Wilks' $\lambda=0.86, \mathrm{~F}[5,132]=4.45, p=0.001$ ), county (Wilks' $\lambda=0.91, \mathrm{~F}[5,132]=2.70, p=0.02)$, and employment status (Wilks' $\lambda=0.89, \mathrm{~F}[5,132]=3.16, p=0.01$ ) were retained for further analyses.

A multivariate regression model was constructed to identify the most robust risk and protective factors of mental health outcomes. The model featured outcomes of depression, suicidal ideation, post-traumatic stress, generalized anxiety, and alcohol use $(r$ s range $=0.30$ to $0.61, p s<0.001$ ). COVID-19 stress and response as well as coping-related factors displaying 
TABLE 4 | Multivariate ${ }^{\star}$ regression model statistics predicting mental health outcomes.

\begin{tabular}{|c|c|c|c|c|}
\hline Predictor & Wilks' $\lambda$ & $F(d f)$ & $p$-value & $\eta_{p}^{2}$ \\
\hline Intercept & 0.09 & $270.30(5,127)$ & $<0.001$ & 0.91 \\
\hline Gender & 0.92 & $2.28(5,127)$ & 0.05 & 0.08 \\
\hline County & 0.99 & $0.40(5,127)$ & 0.85 & 0.01 \\
\hline Employment status & 0.88 & $3.33(5,127)$ & 0.008 & 0.11 \\
\hline COVID-19 stress (other) & 0.82 & $5.59(5,127)$ & $<0.001$ & 0.18 \\
\hline HCRS-10 Stress Response & 0.92 & $2.13(5,127)$ & 0.07 & 0.08 \\
\hline HCRS-10 Emotional Distress & 0.93 & $1.77(5$ 127) & 0.12 & 0.06 \\
\hline HCRS-10 Physical Activity & 0.98 & $0.57(5,127)$ & 0.72 & 0.02 \\
\hline HCRS-10 Use of Alcohol/Drugs & 0.93 & $2.01(5,127)$ & 0.08 & 0.07 \\
\hline HCRS-10 Use of Prescription Medication & 0.94 & $1.71(5,127)$ & 0.14 & 0.06 \\
\hline HCRS-10 Pain & 0.97 & $0.72(5,127)$ & 0.61 & 0.03 \\
\hline HCRS-10 fear/Worry & 0.83 & $5.15(5,127)$ & $<0.001$ & 0.17 \\
\hline HCRS-10 Effort to Cope with Stress & 0.98 & $0.54(5,127)$ & 0.75 & 0.02 \\
\hline Resilience & 0.97 & $0.78(5,127)$ & 0.57 & 0.03 \\
\hline Active coping & 0.89 & $3.17(5,127)$ & 0.01 & 0.11 \\
\hline Venting & 0.83 & $5.01(5,127)$ & $<0.001$ & 0.16 \\
\hline Problem-Focused coping beliefs & 0.91 & $2.51(5,127)$ & 0.03 & 0.09 \\
\hline Thought Stopping Beliefs & 0.98 & $0.48(5,127)$ & 0.79 & 0.02 \\
\hline
\end{tabular}

Bold font denotes significant multivariate predictor.

HCRS-10, Hilgeman Coronavirus Response Scale-10.

"Multivariate analyses allow for inclusion of multiple dependent variables in one model and provide overall omnibus tests for each predictor ${ }^{61}$.

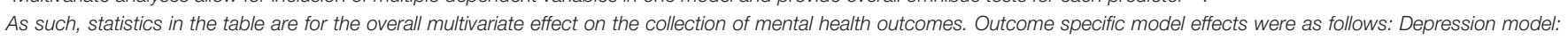

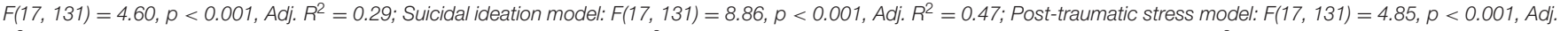
$R^{2}=0.31$; Generalized anxiety model: $F(17,131)=6.88, p<0.001$, Adj. $R^{2}=0.40 ;$ Alcohol use model: $F(17,131)=5.57, p<0.001$, Adj. $R^{2}=0.34$.

significant correlations with two or more mental health outcomes (see Table 1) were included as main effect predictors in the multivariate model. Gender, county, and employment status were demographic covariates. This resulted in a final set of regression model predictors of: gender, employment status, county, COVID-19 Stress (other), stress response, emotional distress, physical activity, use of alcohol/drugs, use of prescription medication, pain, fear/worry, effort to cope with stress, brief resilience, active coping, venting, problem-focused coping beliefs, and thought stopping beliefs. Table 4 contains multivariate test statistics for each predictor; only significant multivariate predictors were inspected as univariate risk or protective factors. Significant multivariate predictors (moderateto-large effects) were employment status, COVD-19 related Stress (other), fear/worry reaction to the pandemic, active coping, venting, and problem-focused coping beliefs. Significant risk and protective factors by mental health outcome were as follows:

(1) Depression model: COVID-19 Stress (other) $(B=0.29$, $\mathrm{se} B=0.12, p=0.01)$; venting $(B=0.23$, $\mathrm{se} B=0.11$, $p=0.04)$; problem-focused coping beliefs $(B=-0.53$, se $B=0.19, p=0.007)$.

(2) Suicidal ideation model: COVID-19 Stress (other) $(B=1.69$, se $B=0.79, p=0.03)$; active coping $(B=-2.69$, se $B=0.82$, $p=0.001)$; venting $(B=2.84$, se $B=0.77, p<0.001)$.

(3) Post-traumatic stress model: Fear/worry response $(B=0.58$, se $B=0.16, p<0.001)$; venting $(B=0.49$, se $B=0.14$, $p$ $=0.001$ ).
(4) Generalized anxiety model: Employed $(B=1.84$, se $B=0.76$, $p=0.02)$; COVID-19 stress (other) $(B=0.67, \mathrm{se} B=0.30$, $p=0.02)$; fear/worry response $(B=1.41$, se $B=0.33$, $p<0.001)$; problem-focused coping beliefs $(B=-1.42$, se $B=0.49, p=0.005)$.

(5) Alcohol use model: COVID-19 stress (other) $(B=0.53$, se $B=0.13, p<0.001)$.

\section{DISCUSSION}

The current study was developed in response to the global call to action of the COVID-19 suicide prevention research collaboration (4). The present study contributes to that call by gaining a picture of stress and mental health among lowincome adults in central NC. First, we examined the prevalence of clinically elevated mental health and suicide risk scores at the height of the pandemic. Next, we assessed how COVID-19 related exposure and responses were associated with mental health and suicide outcomes among adults with lower incomes. Findings from the present study indicate increased needs for mental health care and services in the region. We observed elevated rates of probable risk or need for further evaluation for posttraumatic stress, depression, problematic drinking, generalized anxiety, and suicide. The rates of mental health outcomes in the current sample were elevated compared to pre-pandemic global prevalence rates (5). Additionally, the rates of mental health challenges observed within our sample of NC adults are higher 
than national trends in the CDC report at a similar point in time (36). While measurement differences between the CDC report and our study may explicate varying mental health and substance use rates, it is also possible that the individuals in our low-income NC sample face additional layers of stress that amplify mental health and substance use outcomes. However, given that our rates were obtained via use of very brief screening instruments, further evaluation from licensed healthcare providers would be necessary for formal diagnosis.

A variety of COVID-19 stressors and problematic responses were observed. We observed high rates of COVID-19 illness and exposure. Common stresses resulting from the COVID19 pandemic included job/financial loss, increased home responsibilities, and difficulties with basic necessities (e.g., getting medication). Such stressors ranged from 26 to $47 \%$ of the sample, with higher rates of some stressors among other known persons (see Table 2). Open-ended responses illuminated additional impacts such as decreased socialization and negative emotions. Themes of pandemic-driven financial stress, socialization difficulties, fear of illness, and concerns about mental health are consistent with prior literature (18, 21-23). Finally, the fact that CRS-10 items were positively correlated with many mental health concerns suggests that stress responses may reflect ineffective coping strategies (e.g., physical activity) or additional symptoms of stress reactions to the pandemic (e.g., pain).

The third study aim concerned identifying pandemicrelated risk factors for reduced mental health outcomes. Extending existing literature highlighting the overall severity of fear and worry during the pandemic $(11,62)$, COVID-19 related fear/worry was also a prominent risk factor for posttraumatic stress and anxiety symptoms in particular. Further, our correlational and regression findings suggest that knowing someone else struggling with COVID-19 related stress was more problematic for personal mental health than one's own exposure or stress. This fact is pivotal to understanding the regional effects of the pandemic. Other salient mental health risk factors included a venting coping style (for both symptoms of depression and post-traumatic stress). Consistent with a few national studies $(23,24)$, active coping and problem-focused coping beliefs show some promise as protective factors for depression and suicide risk, respectively, during the pandemic.

\section{Limitations}

This study possesses several clear limitations that temper conclusions and recommendations. Convenience sampling via online methods placed an obvious limitation on who could be reached for the needs assessment. Further, the sample was intentionally restricted by certain demographics, and the racial composition was unclear due to a survey readability concern (see Online Supplement Note). Additionally, the sample size of the current study was small. Moreover, we could not calculate a survey response rate due to absence of the total possible population reached with the study advertisement. Thus, generalizability to the full region is quite restricted. Also, as is often the case with intentionally brief clinical screening instruments (e.g., PHQ-2, COVID-19 stress, Brief
COPE screeners), internal consistency was low for several measures. Psychometric research suggests that a low number of items (four or less) can cause low internal consistency. Low reliability offers a potential explanation of any non-significant findings in the present study because low internal consistency limits the ability to detect correlations with other measures. This limitation does not, however, render the screening tools useless in applied/field public health research. The benefit of short screeners is real world efficiency in identifying persons who may be at risk for a number of clinical conditions or stress coping problems, and to evaluate those relationships among the persons who need assistance. Future research, program evaluation, and follow-up in this area should employ longer clinically-relevant mental health and coping tools. Finally, the online data collection administration may have limited survey access; although many people in urban areas are connected to the Internet, prioritizing populations who may experience financial and technological resource deficits compromised our ability to reach the full scope of community partners. Although we employed a community-engaged strategy, further community partnerships with local faith and other community leaders and agencies can expand additional needs assessments and follow-up public health programming.

\section{Public Health and Research Implications}

Findings from this study suggest possible avenues for a regional public health strategy to address the adverse mental health effects of the pandemic in the Charlotte, NC region. A public health approach should include the following facets. First, given the high positive screening rates for mental health problems, programs should focus on regional investment in virtual training of mental health providers in leading assessment practices and evidence-based therapies to treat mental health and substance use disorders. Such therapeutic approaches may include the Collaborative Assessment and Management of Suicide (CAMS) (63), Dialectical Behavior Therapy (DBT) (64), and CognitiveBehavioral Therapy (CBT) techniques such as Motivational Interviewing and Cognitive Restructuring (65). Given that access to specialty mental health care is limited, particularly for individuals with low incomes, embedding mental health providers in medical settings where COVID-19 diagnostic procedures and heightened stress may be present (e.g., primary care, community health clinics; emergency departments) is critical to reaching those in need of services.

Research also demonstrates efficacy of online alcohol interventions, especially when accompanied by therapeutic principles and person support (66). In light of logistical limitations (e.g., social contact, limited transportation) the pandemic may impose additional impediments on people with lower incomes; therefore, more equitable efforts in the region may promote existing virtual alcohol interventions such as virtual 12-step programs or cognitive-behaviorally based therapies. Selection and implementation of online alcohol interventions should occur in consultation with a qualified substance use expert. Alternatively, mental health service providers may seek to implement online therapy groups for persons with problematic 
substance use in the region; such programs can tackle specific problems identified in this needs assessment.

A variety of community-based responses may further address the stress and mental health implications of the pandemic. For instance, community-based screenings are a common practice to detect persons at risk for various mental health and alcohol use disorders (67). We recommend the widespread use of COVID19 stress and general mental health screening instruments such as those employed in this assessment. They may be implemented in-person or online by partnering with regional agencies, emergency department or primary care facilities, or other non-profit entities. When used online, screening tools should be accompanied by clear instructions on how to reach a provider. Another community-focused strategy is the design of a comprehensive pandemic awareness campaign. Social media, radio, newspaper print, podcast, and other platforms can unify public health messaging such as the importance of remaining socially distanced yet connected, mental health warning signs and benefits of therapy, and free, brief coping skills tools. Additionally, awareness campaign design should employ principles of behavior change theories such as the Theory of Planned Behavior (68). Campaign messages can be augmented by public health educational materials for social media and print distribution via non-profit, academic, and healthcare entities in the region.

Our findings also have implications informing public health research moving forward. As COVID-19 becomes endemic, future areas of study will need to identify suitable assessment tools including standardized domains of data collection to monitor the mental health consequences of adjusting to outbreaks and public health interventions to address COVID-19 over time. We utilized two unpublished inventories developed: The Coronavirus Stress Survey (CSS) and Coronavirus Response Scale (CRS). We selected these tools as they are consistent with large-scale research efforts by a leading national healthcare agency, namely the Department of Veterans Affairs. Our findings provide preliminary data suggesting these tools may be useful for future COVID-19 stress and related research. However, other

\section{REFERENCES}

1. United States Department of Health and Human Services. Determination that a Public Health Emergency Exists. Washington, D.C.: U.S. Department of Health and Human Services. 2020. Available online at: https://www.phe.gov/ emergency/news/healthactions/phe/Pages/2019-nCoV.aspx

2. Johns Hopkins. COVID-19 Data in Motion: Sunday, September 12, 2021. Baltimore, MD: Johns Hopkins University and Medicine Coronavirus Resource a. Available online at: https://coronavirus.jhu.edu

3. Torales J, O’Higgins M, Castaldelli-Maia JM, Ventriglio A. The outbreak of COVID-19 coronavirus and its impact on global mental health. Int J Soc Psychiatry. (2020) 66:317-20. doi: 10.1177/0020764020915212

4. Gunnell D, Appleby L, Arensman E, Hawton K, John A, Kapur N, et al. Suicide risk and prevention during the COVID-19 pandemic. Lancet Psychiatry. (2020) 7:468-71. doi: 10.1016/S2215-0366(20)30171-1

5. Charlson F, van Ommeren M, Flaxman A, Cornett J, Whiteford H, Saxena S. New WHO prevalence estimates of mental disorders in conflict settings: A systematic review and meta-analysis. Lancet. (2019) 394:240-8. doi: 10.1016/S0140-6736(19)30934-1 relevant emerging measures exist, such as the COVID-19 Anxiety Scale, (69) that also warrant further study. Mixed-methods findings from our study highlight a number of possible outcomes for COVID-19 stress, coping, and mental health intervention, and program evaluation research. Namely, COVID-19 stress, coping self-efficacy, and a myriad of mental health domains may be the subject of intervention development and evaluation moving forward.

\section{DATA AVAILABILITY STATEMENT}

A de-identified dataset is available by direct contact of the corresponding author at rcramer4@uncc.edu.

\section{ETHICS STATEMENT}

The current study involved human participants and was reviewed and approved by UNC Charlotte Institutional Review Board. Participants provided informed consent to participate in this study electronically.

\section{AUTHOR CONTRIBUTIONS}

AK, RC, CM, AP, MZ, and JL-R: writing. Y-JH, GG, SS, CW, AK, $\mathrm{RC}$, and AM: data collection. RC, AK, and CW: data analysis. Y-JH, RC, AM, and JL-R: getting funding. RC, MZ, and JL-R: supervision. All authors: editing.

\section{FUNDING}

This work was supported by a generous grant from the Cardinal Innovations Healthcare COVID-19 Relief Fund.

\section{SUPPLEMENTARY MATERIAL}

The Supplementary Material for this article can be found online at: https://www.frontiersin.org/articles/10.3389/fpsyt. 2021.790468/full\#supplementary-material
6. Sareen J, Afifi TO, McMillan KA, Asmundson GJ. Relationship between household income and mental disorders: findings from a populationbased longitudinal study. Arch Gen Psychiatry. (2011) 68:419-27. doi: 10.1001/archgenpsychiatry.2011.15

7. Pfefferbaum B, North CS. Mental health and the Covid-19 pandemic. N Engl J Med. (2020) 383:510-2. doi: 10.1056/NEJMp2008017

8. Sprang G, Silman M. Posttraumatic stress disorder in parents and youth after health-related disasters. Disaster Med Public Health Prep. (2013) 7:105-10. doi: $10.1017 / \mathrm{dmp} .2013 .22$

9. Sim K, Huak Chan Y, Chong PN, Chua HC, Wen Soon S. Psychosocial and coping responses within the community health care setting towards a national outbreak of an infectious disease. J Psychosom Res. (2010) 68:195202. doi: 10.1016/j.jpsychores.2009.04.004

10. Luyt CE, Combes A, Becquemin MH, Beigelman-Aubry C, Hatem S, Brun A, et al. Long-term outcomes of pandemic 2009 influenza A(H1N1)-associated severe ARDS. Chest. (2012) 142:583-92. doi: 10.1378/chest.11-2196

11. Gao J, Zheng P, Jia Y, Chen H, Mao Y, Chen S, et al. Mental health problems and social media exposure during COVID-19 outbreak. PLoS ONE. (2020) 15:e0231924. doi: 10.1371/journal.pone.0231924 
12. Xiang YT, Yang Y, Li W, Zhang L, Zhang Q, Cheung T, et al. Timely mental health care for the 2019 novel coronavirus outbreak is urgently needed. Lancet Psychiat. (2020) 7:228-9. doi: 10.1016/S2215-0366(20)30046-8

13. Daly M, Sutin AR, Robinson E. Longitudinal changes in mental health and the COVID-19 pandemic: evidence from the UK Household Longitudinal Study [published online ahead of print, 2020 Nov 13]. Psychol Med. (2020) 1-10. doi: 10.1017/S003329172000 4432

14. Nochaiwong S, Ruengorn C, Thavorn K, Hutton B, Awiphan R, Phosuya C, et al. Global prevalence of mental health issues among the general population during the coronavirus disease-2019 pandemic: a systematic review and meta-analysis. Sci Rep. (2021) 10173. doi: 10.1038/s41598-02189700-8

15. Calati R, Ferrari C, Brittner M, Oasi S, Olié E, Carvalho AF, et al. Suicidal thoughts and behaviors and social isolation: A narrative review of the literature. J Affect Disord. (2019) 245:653-67. doi: 10.1016/j.jad.2018.11.022

16. Woolf SH, Schoomaker H. Life expectancy and mortality rates in the United States, 1959-2017. JAMA. (2019) 322:1996-2016. doi: 10.1001/jama.2019.16932

17. Zhang Y, Ma ZF. Impact of the COVID-19 pandemic on mental health and quality of life among local residents in liaoning province, china: a cross-sectional study. Int J Environ Res Public Health. (2020) 17:2381. doi: 10.3390/ijerph17072381

18. Son C, Hegde S, Smith A, Wang X, Sasangohar F. Effects of COVID-19 on college students' mental health in the United States: interview survey study. $J$ Med Internet Res. (2020) 22:e21279. doi: 10.2196/21279

19. Leigh-Hunt N, Bagguley D, Bash K, Turner V, Turnball S, Valtora N, et al. An overview of systematic reviews on the public health consequences of social isolation and loneliness. Public Health. (2017) 152:157-71. doi: 10.1016/j.puhe.2017.07.035

20. Marroquín B, Vine V, Morgan R. Mental health during the COVID19 pandemic: Effects of stay-at-home policies, social distancing behavior, and social resources. Psychiatry Res. (2020) 293:113419. doi: 10.1016/j.psychres.2020.113419

21. Holmes EA, O’Connor RC, Perry VH, Tracey I, Wessely S, Arseneault L, et al. Multidisciplinary research priorities for the COVID-19 pandemic: a call for action for mental health science. Lancet Psychiat. (2020) 7:547-60. doi: 10.1016/S2215-0366(20)30168-1

22. Li S, Wang Y, Xue J, Zhao N, Zhu T. The Impact of COVID-19 Epidemic declaration on psychological consequences: a study on active weibo users. Int J Environ Res Public Health. (2020) 17:2032. doi: 10.3390/ijerph17062032

23. Park CL, Russell BS, Fendrich M, Finkelstein-Fox L, Hutchinson M, Becker J. Americans' COVID-19 stress, coping, and adherence to CDC guidelines. J Gen Intern Med. (2020) 35:2296-303. doi: 10.1007/s11606-020-05898-9

24. Umucu E, Lee B. Examining the impact of COVID-19 on stress and coping strategies in individuals with disabilities and chronic conditions. Rehabil Psychol. (2020) 65:193-8. doi: 10.1037/rep0000328

25. Garfin DR, Silver RC, Holman EA. The novel coronavirus (COVID-2019) outbreak: Amplification of public health consequences by media exposure. Health Psychol. (2020) 39:355-7. doi: 10.1037/hea0000875

26. Shigemura J, Ursano RJ, Morganstein JC, Kurosawa M, Benedek DM. Public responses to the novel 2019 coronavirus (2019-nCoV) in Japan: Mental health consequences and target populations. Psychiatry Clin Neurosci. (2020) 74:281-2. doi: 10.1111/pcn. 12988

27. Ettman CK, Cohen GH, Abdalla SM, Sampson L, Trinquart L, Castrucci, BC, et al. Persistent depressive symptoms during COVID-19: a national, population-representative, longitudinal study of U.S. adults [published online ahead of print, 2021 Oct 4]. Lancet Reg Health Am. (2021) 100091. doi: 10.1016/j.lana.2021.100091

28. Belen H. Fear of COVID-19 and mental health: the role of mindfulness in during times of crisis. Int J Ment Health Addict. (2021) 1-12. doi: 10.1007/s11469-020-00470-2

29. Horigian VE, Schmidt RD, Feaster DJ. Loneliness, mental health, and substance use among US young adults during COVID-19. J Psychoactive Drugs. (2021) 53:1-9. doi: 10.1080/02791072.2020.1836435

30. United States Census Bureau. Quick Facts: Charlotte city, North Carolina. Suitland, MD: United States Census Bureau. Available online at: https://www. census.gov/quickfacts/charlottecitynorthcarolina
31. Abedi V, Olulana O, Avula V, Chaudhary D, Khan A, Shahjouei S, et al. Racial, economic and health inequality and COVID-19 infection in the United States. Preprint. medRxiv. (2020) 2020.04.26.20079756. doi: 10.1101/2020.04.26.20079756

32. Vahidy FS, Nicolas JC, Meeks JR, Khan O, Pan A. Jones, SL, et al. Racial and ethnic disparities in SARS-CoV-2 pandemic: analysis of a COVID-19 observational registry for a diverse US metropolitan population. BMJ Open. (2020) 10:e039849. doi: 10.1136/bmjopen-2020-039849

33. Adams ML, Katz DL, Grandpre J. Population-based estimates of chronic conditions affecting risk for complications from coronavirus disease, United States. Emerg Infect Dis. (2020) 26:1831-3. doi: 10.3201/eid2608.200679

34. Khunti K, Singh AK, Pareek M. Hanif W. Is ethnicity linked to incidence or outcomes of covid-19? BMJ. (2020) 369:m1548. doi: 10.1136/bmj. m1548

35. Laurencin CT, McClinton A. The COVID-19 pandemic: a call to action to identify and address racial and ethnic disparities. J Racial Ethn Health Disparities. (2020) 7:398-402. doi: 10.1007/s40615-020-00756-0

36. Czeisler MÉ, Lane RI, Petrosky E, Wiley JF, Christensen A, Njai R, et al. Mental health, substance use, and suicidal ideation during the COVID-19 pandemic - United States, June 24-30, 2020. MMWR Morb Mortal Wkly Rep. (2020) 69:1049-57. doi: 10.15585/mmwr.mm6932al

37. American Public Health Association. COVID-19 guidance for highrisk populations. Washington, D.C: American Public Health Association. Available online at: https://www.apha.org/topics-and-issues/communicabledisease/coronavirus/higher-risk-populations

38. Centers for Disease Control and Prevention. Stress and coping. Atlanta, GA: Centers for Disease Control and Prevention. 2020. Available online at: https:// www.cdc.gov/coronavirus/2019-ncov/daily-life-coping/stress-coping/index. html

39. Carney JK, Maltby HJ, Mackin KA, Maksym ME. Community-academic partnerships: how can communities benefit? Am J Prev Med. (2011) 41:S206S213. doi: 10.1016/j.amepre.2011.05.020

40. Psychology for All. What is Psychology for All? Charlotte, NC: Psychology for All. Available online at: https://www.psychologyforall.org

41. Christ Centered Community Counseling. Charlotte, NC: Christ Centered Community Counseling. Available online at: https://www.c4counseling.com

42. Harvest Center. Charlotte, NC: Harvest Center. Available online at: https:// theharvestcenter.org

43. Care Ring. Charlotte, NC: Care Ring. Available online at: https://www. careringnc.com

44. Time Out Youth. Charlotte, NC: Time Out Youth. Available online at: https:// www.timeoutyouth.org

45. Westside Education Think Tank. Charlotte, NC: Westside Education Think Tank. Available online at: https://www.alignable.com/charlotte-nc/westsideeducation-think-tank

46. UNC Charlotte School of Social Work Field Education. Welcome to Field Education. Charlotte, NC: University of North Carolina Charlotte. Available online at: https://socialwork.uncc.edu/field-education/welcomefield-education

47. McLean CP, Cloitre M. Coronavirus stressor survey. Unpublished Document. (2020).

48. Hilgeman MM, Cramer RJ, Hoch M, Heebner N. The Coronavirus Response Scale-10 (CRS-10). Unpublished Manuscript. (2020).

49. Kroenke K, Spitzer RL, Williams JB. The Patient Health Questionnaire-2: Validity of a two-item depression screener. Med Care. (2003) 41:1284-92. doi: 10.1097/01.MLR.0000093487.78664.3C

50. Spitzer RL, Kroenke K, Williams JB. Löwe, B. A brief measure for assessing generalized anxiety disorder: The GAD-7. Arch Intern Med. (2006) 166:10927. doi: 10.1001/archinte.166.10.1092

51. van Spijker BA, Batterham PJ, Calear AL, Farrer L, Christensen H, Reynolds J, et al. The suicidal ideation attributes scale (SIDAS): Communitybased validation study of a new scale for the measurement of suicidal ideation. Suicide Life Threat Behav. (2014) 44:408-19. doi: 10.1111/sltb. 12084

52. Lang AJ, Wilkins K, Roy-Byrne PP, Golinelli D, Chavira D, Sherbourne C, et al. Abbreviated PTSD Checklist (PCL) as a guide to clinical response. Gen Hosp Psychiatry. (2012) 34:332-8. doi: 10.1016/j.genhosppsych.2012.02.003 
53. Lang AJ, Stein MB. An abbreviated PTSD checklist for use as a screening instrument in primary care. Behav Res Ther. (2005) 43:585-94. doi: 10.1016/j.brat.2004.04.005

54. Spoont M, Arbisi P, Fu S, Greer N, Kehle-Forbes S, Meis L, et al. Screening for post-traumatic stress disorder (PTSD) in primary care: A systematic review. Department of Veterans Affairs Health Services Research \& Development Service Veterans Affairs Evidence-based Synthesis Program Project \#09-009. (2013).

55. Bush K, Kivlahan DR, McDonell MB, Fihn SD, Bradley KA. The AUDIT alcohol consumption questions (AUDIT-C): an effective brief screening test for problem drinking. Ambulatory care quality improvement project (ACQUIP) alcohol use disorders identification test. Arch Intern Med. (1998) 158:1789-95. doi: 10.1001/archinte.158.16.1789

56. Campbell CE, Maisto SA. Validity of the AUDIT-C screen for at-risk drinking among students utilizing university primary care. J Am Coll Health. (2018) 66:774-82. doi: 10.1080/07448481.2018.1453514

57. Bowring AL, Gouillou M, Hellard M, Dietze P. Comparing short versions of the AUDIT in a community-based survey of young people. BMC Public Health. (2013) 13:301. doi: 10.1186/1471-2458-13-301

58. Carver CS. You want to measure coping but your protocol's too long: Consider the brief COPE. Int J Behav Med. (1997) 4:92-100. doi: 10.1207/s15327558ijbm0401_6

59. Chesney MA, Neilands TB, Chambers DB, Taylor JM, Folkman S. A validity and reliability study of the coping self-efficacy scale. Br J Health Psychol. (2006) 11:421-37. doi: 10.1348/135910705X53155

60. Vaishnavi S, Connor K, Davidson JR. An abbreviated version of the Connor-Davidson Resilience Scale (CD-RISC), the CD-RISC2: psychometric properties and applications in psychopharmacological trials. Psychiatry Res. (2007) 152:293-7. doi: 10.1016/j.psychres.2007. 01.006

61. Cohen J, Cohen P, West SG, Aiken LS. Applied Multiple Regression/Correlation Analysis for the Behavioral Sciences. 3rd ed Routledge. (2002).

62. Chidiebere Okechukwu E, Tibaldi L, La Torre G. The impact of COVID19 pandemic on mental health of Nurses. Clin Ter. (2020) 171:e399-400. doi: 10.1016/j.envres.2020.110620

63. Jobes DA. The Collaborative Assessment and Management of Suicidality (CAMS): an evolving evidence-based clinical approach to suicidal risk. Suicide Life Threat Behav. (2012) 42:640-53. doi: 10.1111/j.1943-278X.2012. 00119.x
64. Dineff L, Linehan MM. Dialectical behavior therapy in a nutshell. The California Psycholog. (2001) 34:10-3.

65. Riper H, Andersson G, Hunter SB, de Wit J, Berking M, Cuijpers P. Treatment of comorbid alcohol use disorders and depression with cognitive-behavioural therapy and motivational interviewing: A meta-analysis. Addiction. (2014) 109:394-406. doi: 10.1111/add.12441

66. Riper H, Hoogendoorn A, Cuijpers P, Karyotaki E, Boumparis M, Mira $\mathrm{A}$, et al. Effectiveness and treatment moderators of internet interventions for adult problem drinking: An individual patient data meta-analysis of 19 randomised controlled trials. PLoS Med. (2018) 15:e1002714. doi: 10.1371/journal.pmed.1002714

67. Cramer RJ, Judah MR, Badger NL, Holley AM, Judd S, Peterson M, et al. Suicide on college campuses: A public health framework and case illustration. J Am Coll Health. (2020) 1-8. doi: 10.1080/07448481.2020.173 9053

68. Ajzen I. The theory of planned behavior. Organ Behav Hum Decis Process. (1991) 48:179-211. doi: 10.1016/0749-5978(91)90020-T

69. Chandu VC, Pachava S, Vadapalli V, Marella Y. Development and Initial Validation of the COVID-19 Anxiety Scale. Indian J Public Health. (2020) 64:S201-4. doi: 10.4103/ijph.IJPH_492_20

Conflict of Interest: The authors declare that the research was conducted in the absence of any commercial or financial relationships that could be construed as a potential conflict of interest.

Publisher's Note: All claims expressed in this article are solely those of the authors and do not necessarily represent those of their affiliated organizations, or those of the publisher, the editors and the reviewers. Any product that may be evaluated in this article, or claim that may be made by its manufacturer, is not guaranteed or endorsed by the publisher.

Copyright (c) 2022 Kaniuka, Cramer, Wilsey, Langhinrichsen-Rohling, Mennicke, Patton, Zarwell, McLean, Harris, Sullivan and Gray. This is an open-access article distributed under the terms of the Creative Commons Attribution License (CC BY). The use, distribution or reproduction in other forums is permitted, provided the original author(s) and the copyright owner(s) are credited and that the original publication in this journal is cited, in accordance with accepted academic practice. No use, distribution or reproduction is permitted which does not comply with these terms. 